\title{
Correlation Demodex Folliculorum and Skin Biophysical Parameters
}

\author{
Ts. Sarangua ${ }^{1}$, A. Gurbadam ${ }^{2}$, Ya. Enkhtur ${ }^{3^{*}}$ \\ ${ }^{1}$ Department of Biology and Histology, Health Sciences University of Mongolia, Ulaanbaatar, Mongolia; ${ }^{2}$ Department of Biology \\ and Histology, Health Sciences University of Mongolia, Ulaanbaatar, Mongolia; ${ }^{3}$ Department of Dermatology, Health Sciences Uni- \\ versity of Mongolia, Ulaanbaatar, Mongolia. \\ Email: *enkhtur.hsum@yahoo.com
}

Received July 25 ${ }^{\text {th }}, 2013$; revised August $24^{\text {th }}, 2013$; accepted August $31^{\text {st }}, 2013$

Copyright (C) 2013 Ts. Sarangua et al. This is an open access article distributed under the Creative Commons Attribution License, which permits unrestricted use, distribution, and reproduction in any medium, provided the original work is properly cited.

\begin{abstract}
Purpose: Demodex folliculorum is a transparent mite, $0.3-0.4 \mathrm{~mm}$ long, which asymptomatically parasitizes the human pilosebaceous follicles. D. folliculorum is observed in normal skin with a prevalence of $100 \%$ and a density of $\leq 5$ $\mathrm{D} / \mathrm{cm}^{2}$ in the adult population. Materials and Methods: This study evaluated the differences in $\mathrm{pH}$ on the five facial sites between the two populations with and without $\mathrm{D}$. folliculorum. The relationship between $\mathrm{pH}$ and skin surface oil was analyzed in populations with and without Demodex mites. Mongolian 750 subjects ( 370 males and 380 females) aged $16-84$ years, who lived in Ulaanbaatar city, were enrolled in this study. The eligibility criteria included a minimum age of 16 and no physical and dermatologic illness, no cosmetic. Results: Skin surface oil among the five facial areas differed significantly in both subjects with and without Demodex folliculorum $(\mathrm{P}<0.001)$. When the skin surface oil on the T-zone, U-zone was compared, those on the T-zone were significantly higher than those of the U-zone $(\mathrm{P}<$ $0.001)$ in both populations $(\mathrm{P}<0.01)$. The $\mathrm{pH}$ levels on the five facial sites, and U-zone, T-zone and $\mathrm{MFpH}$ differed significantly in among both subjects with and without Demodex folliculorum $(\mathrm{P}<0.001)$. In both groups, the pH levels of the U-zone were significantly lower than the $\mathrm{pH}$ of the T-zone $(\mathrm{P}<0.001)$. Conclusions: Among the five facial sites in subjects with Demodex folliculorum, there were significant negative correlations between skin surface oil and $\mathrm{pH}$ on the forehead $(\mathrm{p}<0.001, \mathrm{r}=652)$, cheek $(\mathrm{P}<0.001, \mathrm{r}=0.656)$, nose $(\mathrm{P}<0.001, \mathrm{r}=0.754)$, chin $(\mathrm{P}<0.001, \mathrm{r}=0.679)$, and T-zone ( $\mathrm{P}<0.001, \mathrm{r}=0.698)$, MFSE-MFpH $(\mathrm{P}<0.001, \mathrm{r}=0.483)$. In contrast, among the five sites in subjects without Demodex folliculorum, a significantly negative correlation between skin surface oil and $\mathrm{pH}$ was observed on the forehead $(\mathrm{p}<0.001, \mathrm{r}=766)$, cheek $(\mathrm{P}<0.001, \mathrm{r}=0.798)$, nose $(\mathrm{P}<0.001, \mathrm{r}=0.706)$, chin $(\mathrm{P}<0.001, \mathrm{r}=0.823)$, and T-zone $(\mathrm{P}<0.001, \mathrm{r}=0.602)$, U-zone $(\mathrm{P}<0.001, \mathrm{r}=-0.550)$.
\end{abstract}

Keywords: Demodex Folliculorum; Skin Surface Oil; Skin Surface pH; Skin-pH-Meter

\section{Introduction}

Demodex folliculorum is a transparent mite, $0.3-0.4$ $\mathrm{mm}$ long, which asymptomatically parasitizes the human pilosebaceous follicles [1]. D. folliculorum is observed in normal skin with a prevalence of $100 \%$ and a density of $\leq 5 \mathrm{D} / \mathrm{cm}^{2}$ in the adult population $[1,2]$.

Subjects with Demodex folliculorum compared to subjects without Demodex folliculorum have been well established by several researchers. Youn et al. proposed a method to objectively classify facial skin types depending on skin surface oil levels (CL) measured by the Sebumeter ${ }^{\mathbb{B}}[3]$.

${ }^{*}$ Corresponding author.
Skin surface $\mathrm{pH}$ is another noteworthy skin biophysical parameter to associate with Demodex folliculorum because the "acid mantle" (acidic skin surface) is thought to have at least four major important effects on the growth rate of P.acnes, the production rate of exoenzymes and antigens, the stability of exoenzymes, and enzyme activity [4-6]. In addition, a correlation between sebum secretion and $\mathrm{pH}$ has not been researched in these Mongolian populations. This study also evaluated the differences in $\mathrm{pH}$ on the five facial sites between the two populations. Lastly, the relationship between $\mathrm{pH}$ and sebum secretion was analyzed in populations with and without Demodex. These differences should be further examined in future studies in Mongolian youth. 


\section{Materials and Methods}

Mongolian subjects who lived Ulan-Bator (Ulan-Bator city, Mongolia) were asked to participate in the study. The eligibility criteria included a minimum age of 16 and no physical and dermatologic illness, as well as no cosmetic problems. Participants were asked not to wear any cosmetics and not to wash their face within 30 minute prior to the measurements. 750 subjects ( 370 males and 380 females) aged $16-84$ years were enrolled in this study. The subjects were divided by age into 11 groups, i.e., 16 - 21, 22 - 27, 28 - 33, 34 - 39, 40 - 45, 46 - 51, 52 $57,58-63,64-69,70-75$, and $76-84$ years old (classification age group divided by age 5 years old, Provisional guidelines on standard international age classifications, United Nations) [7]. This study evaluated the difference in skin surface $\mathrm{pH}$ and skin surface oil on five facial areas (forehead, nose, chin, right and left cheeks) in a healthy Mongolian population. The relationship between skin surface $\mathrm{pH}$ and skin surface oil were analyzed in each of the two groups. All procedures were performed by the same investigator in a room at a constant temperature $\left(20^{\circ} \mathrm{C}\right)$ and relative humidity $(52 \%)$ from March 2012 until March 2013 to minimize variations caused by different investigators, temperature, humidity, and season. The study protocol was approved by the Human Research and Ethical Committee of Health Sciences University of Mongolia (HSUM). The skin surface oil levels on five different sites of the face: forehead (mid-glabella), nose tip, chin (mental prominence), right and left cheeks (the most prominent area of both zygomas) were measured by the Electronic Skin Analyzer ${ }^{\circledR}$ (PSI Co., Ltd., Korea). Press the Electronic Skin Analy$\operatorname{ser}^{\mathbb{R}}$ sensor smoothly on the skin. Sebum was analyzed from each site on a plastic strip with a constant pressure between 0 and 10. Reclassification of the skin surface oil level was done according to the manufacturer's guidelines based on the sebum measurements, as follows: 1 - 2 $\mu \mathrm{g} / \mathrm{cm}^{-2}$, low oil level; $3-6 \mu \mathrm{g} / \mathrm{cm}^{-2}$, normal oil level; 7 $10 \mu \mathrm{g} / \mathrm{cm}^{-2}$, high oil level. Measurements of the five facial sites were recorded and classified as follows: high sebum secreting zone (T-zone: forehead, nose, and chin) and low sebum secreting zone (U-zone: both cheeks). Reference values for the skin surface oil levels (OL) of the T-zone, U-zone and Mean Facial Sebum Excretions (MFSE) were calculated using the following equations: $\mathrm{T}$-zone $=$ the sum of CL of forehead, nose, and chin $/ 3$ $\mathrm{U}$-zone $=$ the sum of CL of right and left cheeks $/ 2 \mathrm{MFSE}=$ the sum of $\mathrm{CL}$ of forehead, nose, chin, right and left cheeks/5.

The skin surface $\mathrm{pH}$ levels of the five facial areas were measured by the Skincheck-pH-Tester ${ }^{\circledR}$ (Hanna instruments, Germany).

The electrode was washed with distilled water before each measurement. The $\mathrm{pH}$ levels of the T-zone, U-zone, and mean facial $\mathrm{pH}(\mathrm{MFpH})$ were calculated by using the following equations: $\mathrm{T}$-zone $=$ the sum of $\mathrm{pH}$ of forehead, nose, and chin $/ 3 \mathrm{U}$-zone $=$ the sum of $\mathrm{pH}$ of right and left cheeks $/ 2 \mathrm{MFpH}=$ the sum of $\mathrm{pH}$ of forehead, nose, chin, right and left cheeks/5.

The Demodex mite on four different sites of the face: forehead (mid-glabella), nose tip, chin (mental prominence), cheek (the most prominent area of both zygomas) were discovered by as squeeze (fat gland squeezing) and standard skin surface biopsy. The standardized skin surface biopsies (SSSB) technique was a $1 \mathrm{~cm}^{2}$ (10 square $\mathrm{mm})$ red square was drawn on one side of the slide. A droplet of cyanoacrylate glue was dropped on the area where the specimen was to be taken and the reverse side of the slide was pressed on the skin surface. The slide was slowly removed from the skin after $30 \mathrm{~s}$. The samples were brought to Laboratories National Dermatology Center of Mongolia as shortly as possible. Two experienced parasitology specialists under a light microscope at $\times$ $100, \times 200$ and $\times 400$ magnification examined the slides. A subject divided into two groups with and without Demodex folliculorum.

\section{Statistical Analysis}

The differences of skin surface sebum casual levels between subjects with and without D. folliculorum at the five facial sites, T-zone, U-zone, and MFSE were tested by an independent $t$-test after evaluating normality. The same test was used to evaluate the differences of $\mathrm{pH}$ between the two groups at the five facial sites, T-zone, U-zone, and $\mathrm{MFpH}$. The differences of skin surface sebum casual levels among the five facial sites in each population (subjects with and without $\mathrm{D}$. folliculorum) were tested using the ANOVA test after evaluating for normality. These tests were used to assess $\mathrm{pH}$ differences among the five facial sites in each population. The differences in both skin surface sebum casual levels and $\mathrm{pH}$ between the T-zone and U-zone in each population were evaluated using the paired $t$-test. Relationships between sebum casual levels and $\mathrm{pH}$ on the five facial sites, T-zone, U-zone, MFSE, MFpH in each population were tested using the Pearson Correlation Coefficient test. The data were considered significant if $\mathrm{P}<0.05$, unless otherwise noted.

\section{Results}

\subsection{Differences of Skin Surface Sebum Casual Levels between Subjects with and without Demodex Folliculorum}

The five facial areas, specially the nose and T-zone of the subjects with Demodex folliculorum showed significantly higher sebum casual levels, compared to the control population. This difference resulted in statistically higher 
sebum casual levels in the T-zone, and MFSE of the demodectic population than the control population. Skin surface oil levels among the five facial areas differed significantly in both subjects with and without Demodex folliculorum $(\mathrm{P}<0.001)$.

The mean and standard deviation of skin surface oil levels of the four studied sites in the two groups as well as the P-values are listed (Tables $\mathbf{1}$ and $\mathbf{2}$ ).

In subjects with Demodex folliculorum, the skin surface oil level of the chin was significantly different than that of the nose tip. When the skin surface oil levels on the T-zone, U-zone were compared, skin surface oil levels on the T-zone were significantly higher than those of the U-zone $(\mathrm{P}<0.001)$ in both populations $(\mathrm{P}<0.01)$.

\subsection{Differences in $\mathbf{p H}$ between Subjects with and without Demodex Folliculorum}

The $\mathrm{pH}$ levels of the five facial sites, T-zone, U-zone, and MFpH in subjects with and without Demodex folliculorum were compared (Figure 1).

The $\mathrm{pH}$ levels on the five facial sites, and U-zone, T-zone and MFpH differed significantly in among both subjects with and without Demodex folliculorum ("P $<$ 0.001). When the $\mathrm{pH}$ levels on the U-zone, T-zone and $\mathrm{MFpH}$ was compared in both populations $(\mathrm{P}<0.01)$.

The $\mathrm{pH}$ levels between the five facial sites, and Uzone, T-zone and $\mathrm{MFpH}$ differed significantly in both subjects with and without Demodex folliculorum $(\mathrm{P}<$ 0.001). In both groups, the $\mathrm{pH}$ levels of the U-zone were

Table 1. The mean and standard deviation of skin surface oil levels in group with Demodex folliculorum.

\begin{tabular}{ccccc}
\hline & \multicolumn{4}{c}{ with Demodex folliculorum } \\
\cline { 2 - 5 }$\left(\mu \mathrm{g} / \mathrm{cm}^{-2}\right)$ & $\begin{array}{c}\text { Nose tip } \\
8.17 \pm 1.324\end{array}$ & $\begin{array}{c}\text { Chin } \\
7.48 \pm 1.902\end{array}$ & $\begin{array}{c}\text { Forehead } \\
7.3 \pm 1.728\end{array}$ & $\begin{array}{c}\text { Cheek } \\
\end{array}$ \\
\cline { 2 - 5 } & & $\mathrm{P}<0.54 \pm 2.126$ \\
\hline Nose tip & - & - & $\mathrm{P}<0.001$ & $\mathrm{P}<0.001$ \\
Chin & - & - & - & $\mathrm{P}<0.001$ \\
Forehead & - & - & - & - \\
Cheek & - & & & $\mathrm{P}<0.001$ \\
\hline
\end{tabular}

Table 2. The mean and standard deviation of skin surface oil levels in group without Demodex folliculorum.

\begin{tabular}{|c|c|c|c|c|}
\hline \multirow{2}{*}{$\begin{array}{c}\text { Mean } \pm \text { SD } \\
\left(\mu \mathrm{g} / \mathrm{cm}^{-2}\right)\end{array}$} & \multicolumn{4}{|c|}{ without Demodex folliculorum } \\
\hline & $\begin{array}{l}\text { Nose tip } \\
6.03 \pm 1.500\end{array}$ & $\begin{array}{c}\text { Chin } \\
5.05 \pm 2.011\end{array}$ & $\begin{array}{c}\text { Forehead } \\
5.34 \pm 1.689\end{array}$ & $\begin{array}{c}\text { Cheek } \\
4.43 \pm 2.287\end{array}$ \\
\hline Nose tip & - & $\mathrm{P}<0.001$ & $\mathrm{P}<0.001$ & $\mathrm{P}<0.001$ \\
\hline Chin & - & - & $\mathrm{P}<0.001$ & $\mathrm{P}<0.001$ \\
\hline Forehead & - & - & - & $\mathrm{P}<0.001$ \\
\hline Cheek & - & - & - & - \\
\hline
\end{tabular}

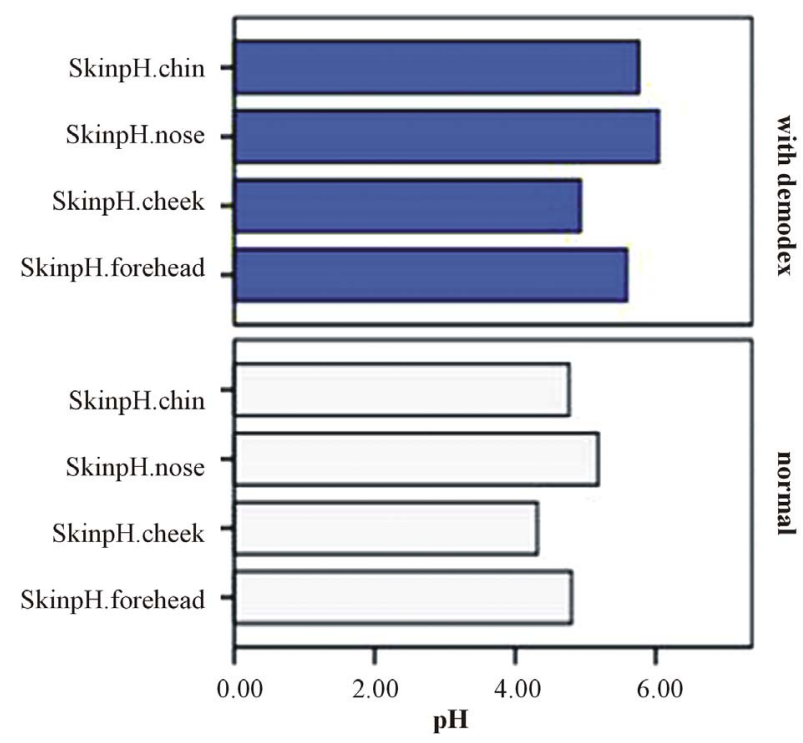

(a)

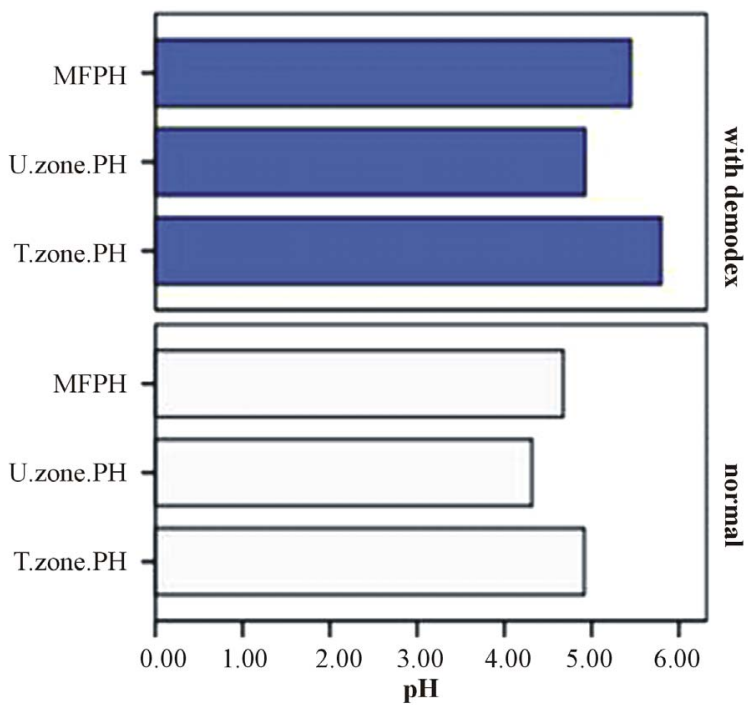

(b)

Figure 1. Difference in pH between subjects with and without Demodex folliculorum. (a): on the cheeks, chin, forehead, and nose tip. (b): on the T-zone, U-zone, and MFpH. Filled bar subjects with Demodex folliculorum, grey bar subjects without Demodex folliculorum, ${ }^{*} \mathbf{P}<0.001$.

significantly lower than the $\mathrm{pH}$ of the $\mathrm{T}$-zone $(\mathrm{P}<$ $0.001)$.

\subsection{Correlations between Skin Surface Oil Levels and pH}

Correlations between skin surface oil levels and $\mathrm{pH}$ on the T-zone and U-zone in subjects with and without Demodex folliculorum are shown (Figure 2).

Among the five facial sites in subjects with Demodex folliculorum, there were significant negative correlations between skin surface oil levels and $\mathrm{pH}$ on the forehead ( $\mathrm{p}$ 


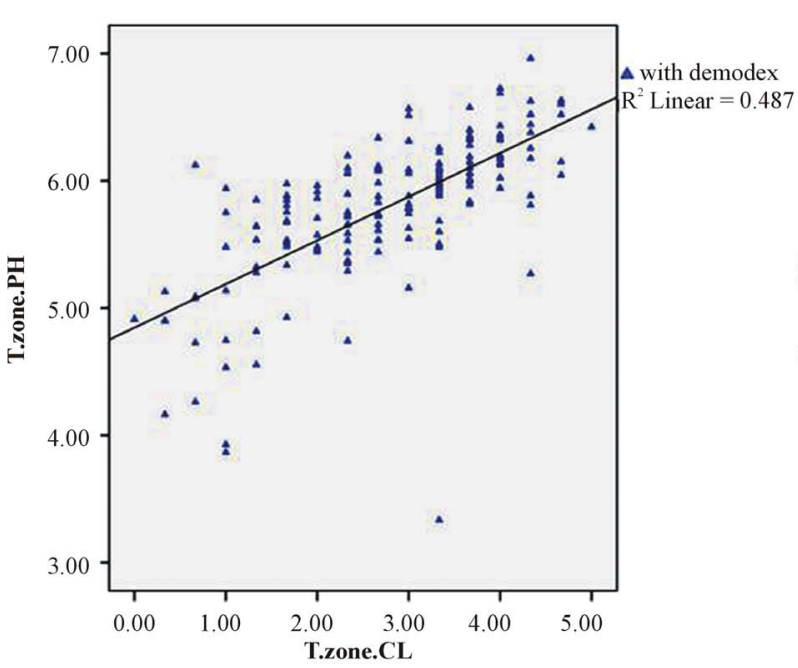

(a1)

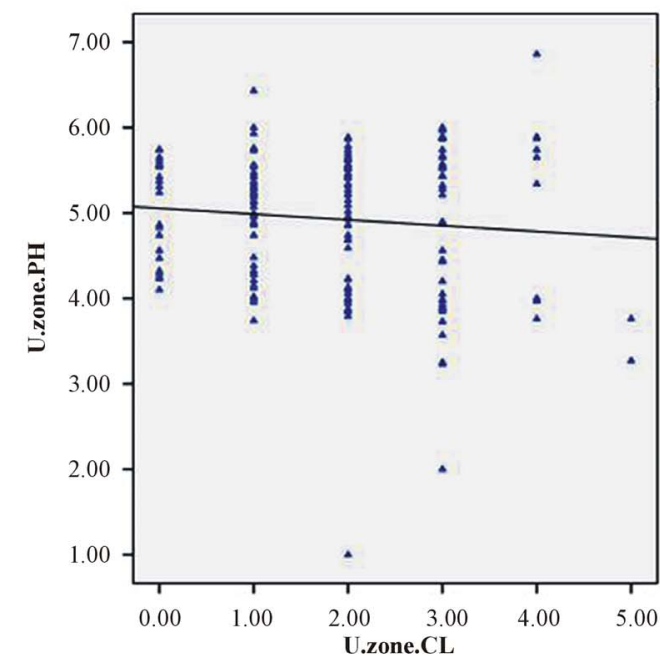

(b1)

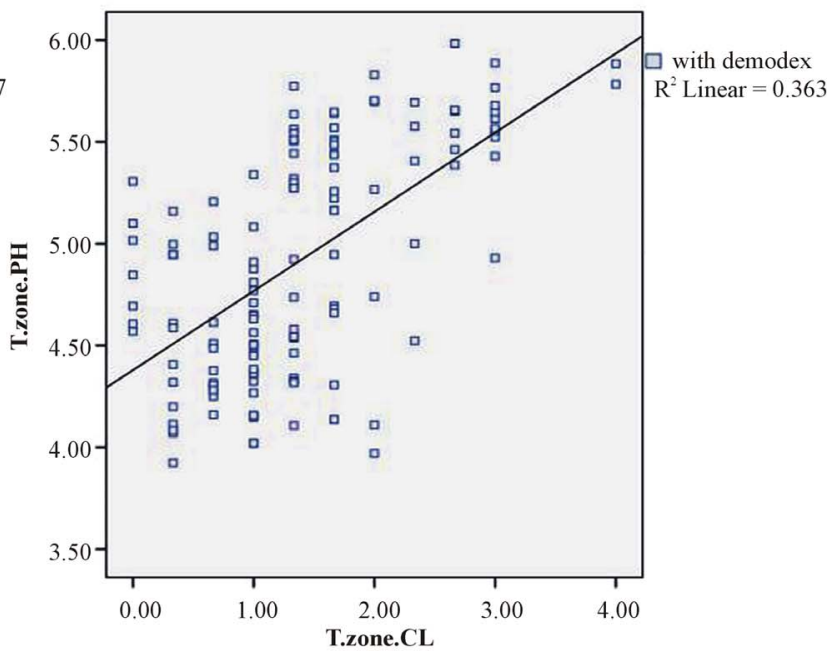

(a2)

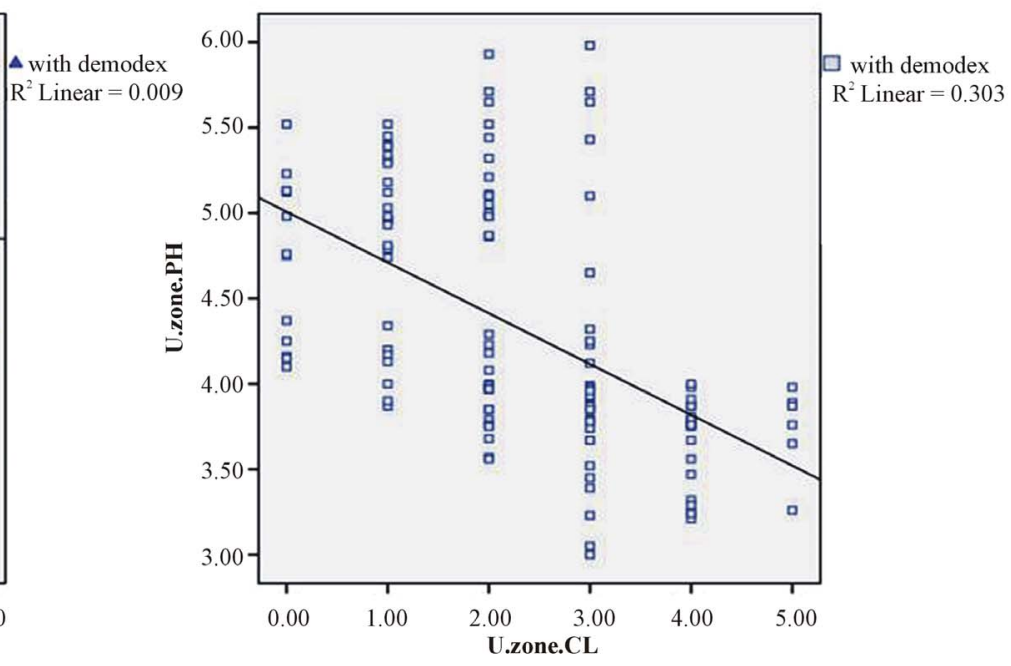

(b2)

Figure 2. Correlations between skin surface sebum casual level and pH. (a1), (a2): T-zone and (b1), (b2): U-zone in subjects with Demodex folliculorum and without Demodex folliculorum. Filled triangle subjects with Demodex folliculorum, empty square subjects without Demodex folliculorum.

$<0.001, \mathrm{r}=652)$, cheek $(\mathrm{P}<0.001, \mathrm{r}=0.656)$, nose $(\mathrm{P}<$ $0.001, \mathrm{r}=0.754)$, chin $(\mathrm{P}<0.001, \mathrm{r}=0.679)$, and $\mathrm{T}$-zone $(\mathrm{P}<0.001, \mathrm{r}=0.698)$, MFSE-MFpH $(\mathrm{P}<0.001, \mathrm{r}=$ $0.483)$.

In contrast, among the five sites in subjects without Demodex folliculorum, a significant negative correlations between skin surface oil levels and $\mathrm{pH}$ was observed on the forehead $(\mathrm{p}<0.001, \mathrm{r}=766)$, cheek $(\mathrm{P}<$ $0.001, \mathrm{r}=0.798)$, nose $(\mathrm{P}<0.001, \mathrm{r}=0.706)$, chin $(\mathrm{P}<$ $0.001, \mathrm{r}=0.823)$, and T-zone $(\mathrm{P}<0.001, \mathrm{r}=0.602)$, $\mathrm{U}-$ zone $(\mathrm{P}<0.001, \mathrm{r}=-0.550)$.

Demodex mites was explored $(\mathrm{p}<0.03)$ by 47.1 percent of the healthy populations. The Demodex mite was explored: 62.2 percent at people aged 16 - 20, 55.6 percent at people aged then up $70(\mathrm{r}=0.106, \mathrm{p}<0.02)$ (Table 3).

\section{Discussion}

In this study, the skin surface sebum casual levels on the nose of the population with Demodex folliculorum were significantly higher than those of the control population, which resulted in statistically higher skin surface sebum casual levels on the T-zone and MFSE of the population with Demodex folliculorum, compared to the control population. These results are similar to the results of other studies, showing that the acne is more intense in subjects with Demodex folliculorum. However, there is a difference between this study and previously published studies with regard to sebum secretion parameters and facial areas which showed a significant difference. Pierard et al. demonstrated different overall sebum excretion rates between subjects with and without acne when they mea- 
Table 3. Correlation Demodex Folliculorum and study groups for age.

\begin{tabular}{|c|c|c|c|c|c|}
\hline \multirow{2}{*}{$\begin{array}{l}\text { Age groups } \\
\quad \text { (years) }\end{array}$} & \multirow{2}{*}{$\mathrm{n}$} & \multicolumn{2}{|c|}{ With Demodex Folliculorum } & \multicolumn{2}{|c|}{ Without Demodex Folliculorum } \\
\hline & & $n$ & $\%$ & $n$ & $\%$ \\
\hline $16-20$ & 74 & 46 & 62.1 & 28 & 37.9 \\
\hline $21-24$ & 56 & 26 & 46.4 & 30 & 53.6 \\
\hline $25-29$ & 64 & 38 & 53.6 & 26 & 46.4 \\
\hline $30-34$ & 42 & 17 & 40.5 & 25 & 59.5 \\
\hline $35-39$ & 71 & 26 & 36.6 & 45 & 63.4 \\
\hline $40-44$ & 69 & 23 & 33.3 & 46 & 66.7 \\
\hline $45-49$ & 56 & 25 & 44.7 & 31 & 55.3 \\
\hline $50-54$ & 56 & 19 & 34 & 37 & 66 \\
\hline $55-59$ & 72 & 32 & 44.5 & 40 & 55.5 \\
\hline $60-64$ & 45 & 26 & 57.8 & 19 & 42.2 \\
\hline $65-69$ & 55 & 25 & 45.5 & 30 & 54.5 \\
\hline $70-84$ & 90 & 50 & 55.6 & 40 & 44.4 \\
\hline Total & 750 & 353 & 47.1 & 397 & 52.9 \\
\hline
\end{tabular}

$\mathrm{n}$-number of subjects.

sured sebum secretion of foreheads using Lipometer ${ }^{\circledR}$, a photometric technique [7]. Pierard-Franchimont et al. also demonstrated significant difference of the total sebum excretion on the forehead between subjects with moderate acne and subjects without acne, whereas it did not differ between subjects with mild acne and subjects without acne [8].

The $\mathrm{pH}$ levels among the five facial sites, and U-zone, T-zone and $\mathrm{MFpH}$ differed significantly in both subjects with and without Demodex folliculorum $(\mathrm{P}<0.001)$. This may be due to the enlargement production pilosebaceous glands.

However, there are several studies showing the negative effects of elevated $\mathrm{pH}$ on the skin. In the study of Wendling et al., age did not correlate with skin $\mathrm{pH}[5,6$, 9-11]. In addition, Zlotogorski showed no difference in $\mathrm{pH}$ between the two genders [12].

The $\mathrm{pH}$ among the five studied sites differed significantly in both subjects with Demodex folliculorum and subjects without Demodex folliculorum $(\mathrm{P}<0.001)$. In fact, there is a general consensus in distinguishing facial areas into the T-zone and U-zone depending on sebum secretion. The difference in sebum secretion among different facial areas has been well-documented [13-17]. This study also showed that sebum casual levels of the T-zone were significantly higher than those of the Uzone in each population ( $\mathrm{P}<0.001)$. This may be associated with the difference in sebum secretion due in part to significant negative correlations between sebum casual levels and $\mathrm{pH}$, which were also seen in this study. These differences should be further examined in future studies by young Mongolian investigators.

\section{Conclusion}

The sebum casual levels of the nose in the with Demodex folliculorum population were significantly higher than those of the without Demodex folliculorum population, which resulted in statistically higher sebum casual levels in the T-zone and MFSE of the population with Demodex folliculorum, compared to the control population. The $\mathrm{pH}$ of the five studied areas significantly differed between two groups. The $\mathrm{pH}$ distribution among the facial five studied sites differed significantly in both subjects with Demodex folliculorum and subjects without Demodex folliculorum. The negative correlation between sebum casual levels and $\mathrm{pH}$ was statistically significant in the five studied areas and T-zone, MFSE-MFpH.

\section{Acknowledgements}

I strongly appreciate to everyone that helped my research work. Special thanks to my supervisors Prof. Gurbadam A. PhD in biology and Ass. Prof. Enkhtur Ya. MD, PhD in dermatology, HSUM. Also let me express my gratitude to all the colleagues, Department of Biology and Histology, School of Bio-Medicine, Department of Dermatology, School of Medicine, HSUM, and National Dermatology Center of Mongolia. Allow me to wish all 
above mentioned associates the best in their further researches and long lasting happy life.

\section{REFERENCES}

[1] F. Forton and B. Seys, "Density of Demodex Folliculorum in Rosacea: A Case Control Study Using Standardized Skin Surface Biopsy," British Journal Dermatology, Vol. 128, No. 6, 1993, pp. 650-659. doi:10.1111/j.1365-2133.1993.tb00261.x

[2] E. Bonnar, P. Eustace and F. C. Powell, "The Demodex Mite Population in Rosacea," Journal American Academy of Dermatology, Vol. 28, No. 3, 1993, pp. 443-448. doi:10.1016/0190-9622(93)70065-2

[3] S. W. Youn, S. J. Kim, I. A. Hwang and K. C. Park, "Evaluation of Facial Skin Type by Sebum Secretion: Discrepancies between Subjective Descriptions and Sebum Secretion," Skin Research and Technology, Vol. 8, No. 3, 2002, pp. 168-172. doi:10.1034/j.1600-0846.2002.10320.x

[4] J. Greenman, "Follicular $\mathrm{pH}$ and the Development of Acne," International Journal of Dermatology, Vol. 20, No. 10, 1981, pp. 656-658. doi:10.1111/j.1365-4362.1981.tb00398.x

[5] J. Greenman, K. T. Holland and W. J. Cunliffe, "Effects of $\mathrm{pH}$ on Biomass, Maximum Specific Growth Rate and Extracellular Enzyme Production by Three Species of Cutaneous Propionibacteria Grown in Continuous Culture," Journal of Genetic and Microbiology, Vol. 129, No. 5, 1983, pp. 1301-1307.

[6] J. P. Hachem, D. Crumrine, J. Fluhr, B. E. Brown, K. R. Feingold and P. M. Elias, "pH Directly Regulates Epidermal Permeability Barrier Homeostasis, and Stratum Corneum Integrity/Cohesion," Journal of Investigative Dermatology, Vol. 121, No. 2, 2003, pp. 345-353. doi:10.1046/j.1523-1747.2003.12365.x

[7] Provisional Guidelines on Standard International Age Classifications, "Recommended Standard International Age Classifications for Demographic, Social and Related Economic Data," United Nations, New York, 1982, pp. 5-39.

[8] G. E. Pierard, C. Pierard-Franchimont and T. Le, "Seborrhoea in Acne-Prone and Acne-Free Patients," Dermatologica, Vol. 175, No. 1, 1987, pp. 5-9. doi:10.1159/000248774

[9] C. Pierard-Franchimont, G. E. Pierard, D. Saint-Leger, J.
L. Leveque and A. M. Kligman, "Comparison of the Kinetics of Sebum Secretion in Young Women with and without Acne," Dermatologica, Vol. 183, No. 2, 1991, pp. 120-122. doi: $10.1159 / 000247650$

[10] K. T. Holland, J. Greenman and W. J. Cunliffe, "Growth of Cutaneous Propionibacteria on Synthetic Medium; Growth Yields and Exoenzyme Production," Journal of Applied Bacteriology, Vol. 47, No. 3, 1979, pp. 383-394. doi:10.1111/j.1365-2672.1979.tb01198.x

[11] H. C. Korting, M. Kerscher, M. Schafer-Korting and U. Berchtenbreiter, "Influence of Topical Erythromycin Preparations for Acne Vulgaris on Skin Surface pH," Clinical Investigation, Vol. 71, No. 8, 1993, pp. 644-648.

[12] P. A. Wendling and G. Dell'Acqua, "Skin Biophysical Properties of a Population Living in Valias, Switzerland," Skin Research and Technology, Vol. 9, No. 4, 2003, pp. 331-338. doi:10.1034/j.1600-0846.2003.00040.x

[13] A. Zlotogorski, "Distribution of Skin Surface pH on the Forehead and Cheek of Adults," Archives of Dermatological Research, Vol. 279, No. 6, 1987, pp. 398-401. doi:10.1007/BF00412626

[14] I. Le Fur, S. Lopez, F. Morizot, C. Guinnot and E. Tschachler, "Comparison of Cheek and Forehead Regions by Bioengineering Methods in Women with Different Self-Reported Cosmetic Skin Types," Skin Research and Technology, Vol. 5, No. 3, 1999, pp. 182-188. doi:10.1111/j.1600-0846.1999.tb00129.x

[15] S. Lopez, I. Le Fur, F. Morizot, G. Heuvin, C. Guinot and E. Tschachler, "Transepidermal Water Loss, Temperature and Sebum Levels on Women's Facial Skin Follow Characteristic Patterns," Skin Research and Technology, Vol. 6, No. 1, 2000, pp. 31-36. doi:10.1034/j.1600-0846.2000.006001031.x

[16] S. G. Park, Y. D. Kim, J. J. Kim and S. H. Kang, "Two Possible Classifications of Facial Skin Type by Two Parameters in Korean Women: Sebum Excretion Rate (SER) and Skin Surface Relief (SSR)," Skin Research and Technology, Vol. 5, No. 3, 1999, pp. 189-194. doi:10.1111/j.1600-0846.1999.tb00130.x

[17] S. W. Youn, S. J. Kim, I. A. Hwang and K. C. Park, "Evaluation of Facial Skin Type by Sebum Secretion: Discrepancies between Subjective Descriptions and Sebum Secretion," Skin Research and Technology, Vol. 8, No. 3, 2002, pp. 168-172. doi:10.1034/j.1600-0846.2002.10320.x 